\title{
TITLE:
}

\section{Dielectric dispersion of erythrocyte ghosts}

$\operatorname{AUTHOR}(\mathrm{S})$ :

Asami, $\mathrm{K}$

\section{CITATION:}

Asami, K. Dielectric dispersion of erythrocyte ghosts. PHYSICAL REVIEW E 2006, 73(5): 052903.

ISSUE DATE:

2006-05

URL:

http://hdl.handle.net/2433/50363

RIGHT:

Copyright 2006 American Physical Society 
PHYSICAL REVIEW E 73, 052903 (2006)

\title{
Dielectric dispersion of erythrocyte ghosts
}

\author{
Koji Asami \\ Institute for Chemical Research, Kyoto University, Uji, Kyoto 611-0011, Japan
}

(Received 31 January 2006; published 31 May 2006)

\begin{abstract}
The underlying mechanism of the low-frequency dielectric dispersion, called the $\alpha$ dispersion, of osmotically lysed erythrocytes (erythrocyte ghosts) has remained open since its finding [H. P. Schwan and E. L. Carstensen, Science 125, 985 (1957)]. The $\alpha$ dispersion is peculiar to erythrocyte ghosts and has never been observed for intact erythrocytes. Numerical calculation based on interfacial polarization revealed that the $\alpha$ dispersion is due to the presence of a hole of about $30 \mathrm{~nm}$ in radius in the plasma membrane.
\end{abstract}

DOI: 10.1103/PhysRevE.73.052903

PACS number(s): 87.17.- d, 77.22.Gm, 02.70.Bf

Biological cells and tissues are polarized by an electric field. The polarization includes different kinds of processes with different time constants. Therefore, the effective permittivity of biological cells and tissues shows dielectric dispersions in different frequency regions of the applied ac field: $\alpha$, $\beta$, and $\gamma$ dispersions in order of increasing frequency [1-3]. The $\gamma$ dispersion is due to orientation of water molecules. The $\beta$ dispersion that usually appears at frequencies between $10 \mathrm{kHz}$ and $100 \mathrm{MHz}$ is well interpreted in terms of the Maxwell-Wagner effect or interfacial polarization, which is due to the accumulation of charges at the interfaces between the membrane and the aqueous phases. Several possible mechanisms responsible for the $\alpha$ dispersion at frequencies below about $10 \mathrm{kHz}$ have been proposed. These are related to the gating of ion permeation in excitable membranes [4], intracellular membrane systems connecting with the plasma membrane such as the transverse tubular system in muscle cells [5], and the displacement of counterions around charged cell surfaces [6]. The $\alpha$ dispersion, however, is still not completely understood because of the structural complexities of cells and tissues and technical difficulties in measurement at frequencies below $1 \mathrm{kHz}$ due to electrode polarization.

In 1957, Schwan and Carstensen [7] reported that osmotically lysed bovine erythrocytes (called ghosts) showed " $\alpha$ dispersion" at frequencies below $10 \mathrm{kHz}$ in addition to the $\beta$ dispersion of a characteristic frequency of about $2 \mathrm{MHz}$. The $\alpha$ dispersion has about half the intensity of the $\beta$ dispersion and has never been found for intact erythrocytes. Ghosts are simple spherical cells with the well defined cytoplasm having the same electrical properties as the external medium. The $\beta$ dispersion of ghosts was unequivocally interpreted by interfacial polarization theories with a single-shell model, i.e., a conductive sphere covered with a less conducting shell. Theoretical analysis of the $\beta$ dispersion revealed that the membrane capacitance was almost the same as that of intact erythrocytes and that the membrane still remained in a low conductive state $[7,8]$. In contrast to the $\beta$ dispersion, no clear explanation has been obtained for the polarization mechanism of the $\alpha$ dispersion; the possible mechanisms described above are unlikely for ghosts.

Osmotic lysis forms holes in the erythrocyte membrane, which freely pass large molecules such as hemoglobin. The number of holes per cell and their size were assessed by the efflux kinetics of probe molecules of various sizes, suggesting typically a single hole of $10-20 \mathrm{~nm}$ in radius [9]. The closure of the hole depends on the composition of electrolyte solutions and the incubation temperature. If a hole remains in the ghost membrane, what kinds of effects are expected on the dielectric spectrum of the ghost suspension? This question is important not only for analyzing the $\beta$ dispersion precisely but also for seeking hints on understanding the $\alpha$ dispersion.

If the membrane has no hole, dielectric dispersion of spherical cells can be simply simulated by the theories based on the single-shell model: a spherical cytoplasm (of relative permittivity $\varepsilon_{c}$ and conductivity $\kappa_{c}$ ) covered with a membrane (of $\varepsilon_{m}$ and $\kappa_{m}$ ) in a continuous medium (of $\varepsilon_{a}$ and $\kappa_{a}$ ). Pauly and Schwan [10] demonstrated that the dielectric dispersion of the model includes two relaxation terms in general cases. Since biological cells meet the conditions that the shell thickness $d_{m}$ is much smaller than the sphere radius $R$ and $\kappa_{m} \ll \kappa_{a} \approx \kappa_{c}$, the intensity of the high-frequency relaxation term becomes negligibly small, and thus the dielectric dispersion is approximately represented by one relaxation term. Such an analytical approach, however, is not applicable to a cell model with a hole, and therefore a numerical technique is required.

In a previous paper [11], a three-dimensional finite difference method has been developed to calculate the admittance of a parallel plate capacitor including a cell in a continuous phase. The same method was applied to a cell model with a hole. A cubic lattice with $50 \times 50 \times 50$ lattice points was used for the system. Each lattice point has a cubic element, which is assigned to either the cell interior or the external medium. The boundary between the cell interior and the external medium has a membrane except for the area of the hole. The cell interior, the membrane, and the external medium are all assumed to be isotropic materials having frequencyindependent permittivity and conductivity. The method yields the electric potential distribution in the system by solving the Laplace equation numerically under boundary conditions, viz., an electric potential difference is given between the top and the bottom, and current density is zero at the four sides. In the method, boundary conditions between different materials in the system always hold and tangential current in the membrane is ignored for its negligibly small thickness. The calculation was performed at frequencies below $100 \mathrm{MHz}$ where the quasielectrostatic approximation is applicable. From the electric potential distribution, we can calculate the admittance of the system, which is simply converted into the effective complex permittivity. 


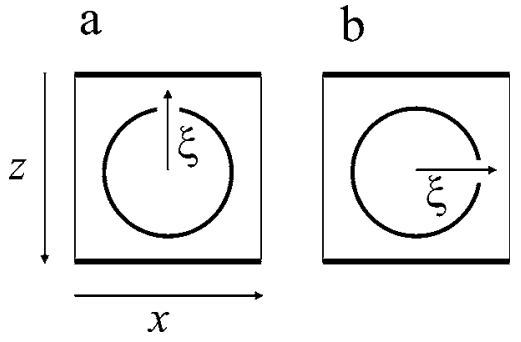

FIG. 1. Cross sections of a parallel plate capacitor of cubic form including a spherical cell with a hole in (a) parallel and (b) perpendicular orientations. The $\xi$ axis is the axis of the cell model, through the centers of the sphere and the hole, being (a) parallel and (b) perpendicular to the electric field. A pair of horizontal bars indicates the plate electrodes. The $z$ and $x$ axes of the system are indicated in (a).

Since the spherical cell model with a hole is symmetric with respect to the $\xi$ axis running through the centers of the sphere and the hole (Fig. 1), its polarization has two components parallel and perpendicular to the $\xi$ axis; anisotropic dielectric properties are expected. Hence, dielectric spectra were calculated when the $\xi$ axis is parallel and perpendicular to the electric field, which arrangements are here termed the parallel and the perpendicular orientations, respectively (Fig. $1)$. The parameter values used for the calculations were relevant to erythrocyte ghost suspensions: $\kappa_{a}=\kappa_{c}=0.25 \mathrm{~S} / \mathrm{m}$, $\varepsilon_{a}=\varepsilon_{c}=80, \varepsilon_{m}=5, \kappa_{m}=10^{-7} \mathrm{~S} / \mathrm{m}, d_{m}=5 \mathrm{~nm}, R=2.5 \mu \mathrm{m}(R$ is the equivalent radius calculated from the surface area of the cell model) and the volume fraction of the cell in the system is 0.2

Figure 2 shows the dielectric spectra calculated for a cell without and with a hole of varying equivalent radius $R_{h}$ as $30,60,140$, and $290 \mathrm{~nm}$. The cell without hole showed di-

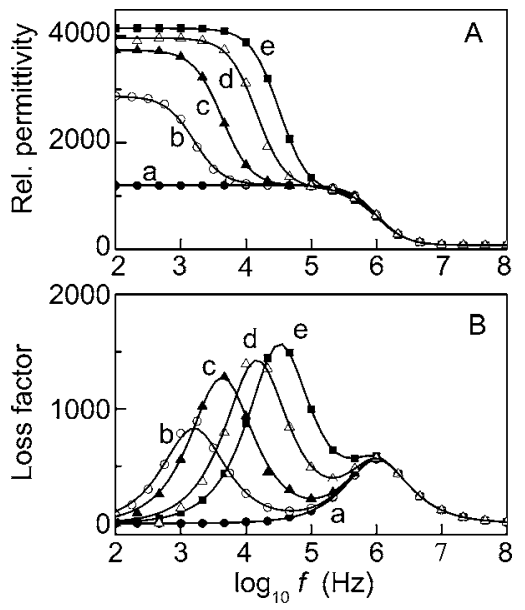

FIG. 2. Dielectric spectra calculated for the spherical cell with and without a hole. (A) The relative permittivity and (B) the loss factor are plotted against the frequency of the applied ac field. The loss factor was calculated by subtracting the contribution of dc conductivity. Curve $a$ in both (A) and (B) correspond to spectra for the cell without hole and the cell with a hole of 30-290 nm in radius in the perpendicular orientation. Curves $b-e$ refer to the cell with a hole of 30,60, 140, and $290 \mathrm{~nm}$, respectively, in radius in the parallel orientation.

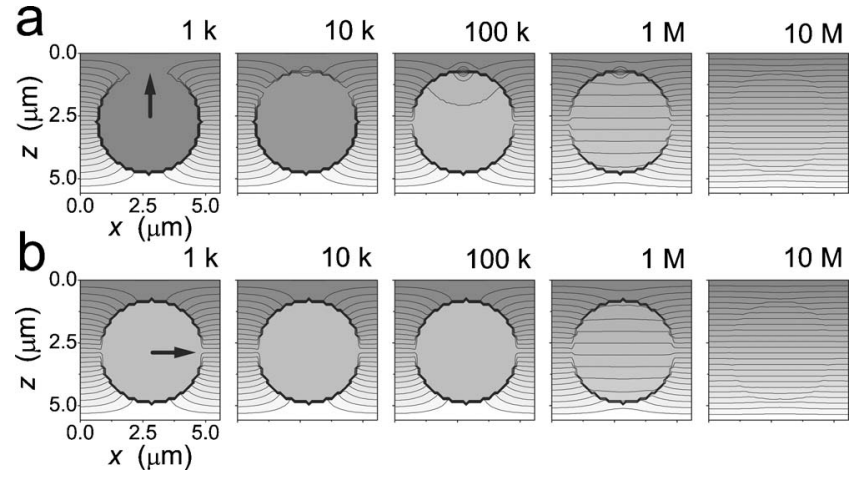

FIG. 3. Electric potential distributions in a capacitor including a cell with a hole in (a) the parallel and (b) perpendicular orientations. Contour plots of the electric potential are shown at cross sections through the centers of the sphere and the hole. The frequency of the applied ac field is indicated in each panel. Arrows in the panels for $1 \mathrm{kHz}$ indicate the position of the hole of $140 \mathrm{~nm}$ in radius. The top and bottom horizontal lines in each panel correspond to the highand low-potential electrodes, respectively.

electric relaxation of a characteristic frequency of about $1 \mathrm{MHz}$. The dielectric spectra for the perpendicular orientation were all the same as that of the cell without hole, irrespective of $R_{h}$. In the parallel orientation, however, dielectric relaxation appeared at frequencies below $100 \mathrm{kHz}$ in addition to the high-frequency relaxation. Both the intensity $\Delta \varepsilon_{1}$ and characteristic frequency $f_{c 1}$ of the low-frequency relaxation depended on $R_{h}$, whereas those of the high-frequency relaxation $\left(\Delta \varepsilon_{2}\right.$ and $\left.f_{c 2}\right)$ were independent of $R_{h}$.

With $R_{h}=30 \mathrm{~nm}$, the intensities and characteristic frequencies of the dielectric relaxation terms were $\Delta \varepsilon_{1}=1660$, $f_{c 1}=1.6 \mathrm{kHz}, \Delta \varepsilon_{2}=1130$, and $f_{c 2}=1.0 \mathrm{MHz}$ for the parallel orientation [curve $b$ in Figs. 2(A) and 2(B)], and those were $\Delta \varepsilon_{2}=1130$ and $f_{c 2}=1.0 \mathrm{MHz}$ for the perpendicular orientation [curve $a$ in Figs. 2(A) and 2(B)]. The values of $f_{c 1}$ and $f_{c 2}$ are, respectively, very close to those of the $\alpha$ dispersion $\left(f_{c 1}=1.7 \mathrm{kHz}\right)$ and the $\beta$ dispersion $\left(f_{c 2}=2 \mathrm{MHz}\right)$ of ghost suspensions [7]. If we assume a random orientation distribution which introduces the factor $1 / 3$ during the ensemble average, the intensity of the low-frequency relaxation $\Delta \bar{\varepsilon}_{1}$ becomes $\Delta \varepsilon_{1} / 3=550$, and that of the high-frequency relaxation $\Delta \bar{\varepsilon}_{2}$ is equal to $\Delta \varepsilon_{2}=1130$ because $\Delta \varepsilon_{2}$ is independent of the orientation. The ratio $\Delta \bar{\varepsilon}_{1} / \Delta \bar{\varepsilon}_{2}$ becomes about 0.5 , being also in good agreement with that obtained for ghost suspensions [7].

The dielectric responses were different between the parallel and perpendicular orientations at low frequencies. To understand the difference, we examine the electric potential distributions in the models corresponding to curves a and $\mathrm{d}$ in Fig. 2. Figure 3 shows the contour plots of the electric potential at the cross sections in the parallel and perpendicular orientations (see Fig. 1). There are similar potential distributions above $100 \mathrm{kHz}$, irrespective of the orientation, viz., the cell interior has almost the same potential value at $100 \mathrm{kHz}$ and the cell is electrically invisible at $10 \mathrm{MHz}$ because the membrane is short-circuited. On the other hand, marked differences in the potential distribution are found between the two orientations below $10 \mathrm{kHz}$. At $1 \mathrm{kHz}$, the cell 
interior in the parallel orientation has a constant potential of which value is equal to that at the position of the hole; there is no potential difference at the top membrane near the hole but a large potential difference at the bottom membrane. Since the potential drop at the membrane indicates that the membrane is polarized and serves as a capacitor, at $1 \mathrm{kHz}$, the larger portion of the membrane accumulates more charges in the parallel orientation than in the perpendicular one. This may lead to a considerable increase in the effective permittivity of the system in the parallel orientation at frequencies below $10 \mathrm{kHz}$.

Does the low-frequency dispersion (the $\alpha$ dispersion) result from the presence of only one hole? When many holes uniformly distribute in the cell membrane, a single-shell model with a lossy shell can represent such a cell. Theories for the model predict a decrease in the relaxation intensity of the $\beta$ dispersion with an increase in the membrane conductivity but never the occurrence of the $\alpha$ dispersion. For a spherical cell with two holes located at its opposite poles, which is a model for a cell treated by high-voltage pulses (namely, an electroporated cell), numerical simulations did not indicate the $\alpha$ dispersion although the intensity of the $\beta$ dispersion decreased with an increase in the size of the hole. When two holes were located in one of the hemispheres of the cell, however, the $\alpha$ dispersion again appeared. These results do not necessarily rule out the presence of more than one hole in a ghost, but the localization of holes is at least required for evoking the $\alpha$ dispersion.

In conclusion, the $\alpha$ dispersion of erythrocyte ghosts can be interpreted in terms of interfacial polarization using a cell model with a hole in the membrane, without considering another polarization process. The $R_{h}$ value that provided the best simulation for the dielectric spectrum found by Schwan and Carstensen [7] was consistent with that estimated by solute efflux kinetics [9]. Thus, the $\alpha$ dispersion would enable us to estimate the size of the hole in ghosts but also to study resealing of holes in damaged cells [12].

I thank Dr. K. Sekine for his critical reading of this manuscript and helpful discussions.
[1] H. P. Schwan, in Advances in Biological and Medical Physics, edited by J. H. Lawrence and C. A. Tobias (Academic Press, New York, 1957), Vol. 5, p. 147.

[2] H. P. Schwan, in The Biophysical Approach to Excitable Systems, edited by W. S. Adelman and D. Goldman (Plenum Press, New York, 1981), p. 3.

[3] K. R. Foster and H. P. Schwan, in Handbook of Biological Effects of Electromagnetic Fields, edited by C. Polk and E. Postow (CRC Press, Boca Raton, 1986), p. 27.

[4] H. M. Fishman, D. Poussart, L. E. Moore, and E. Siebenga, J. Membr. Biol. 32, 255 (1977); 50, 43 (1979).

[5] G. Falk and P. Fat, Proc. R. Soc. London, Ser. B 160, 69
(1964).

[6] G. Schwarz, J. Phys. Chem. 66, 2636 (1962); C. W. Einolf and E. L. Carstensen, ibid. 75, 1091 (1971).

[7] H. P. Schwan and E. L. Carstensen, Science 125, 985 (1957).

[8] H. Kaneko, K. Asami, and T. Hanai, Colloid Polym. Sci. 269, 1039 (1991).

[9] M. R. Lieber and T. L. Steck, J. Biol. Chem. 257, 11651 (1982); 257, 11660 (1982).

[10] H. Pauly and H. P. Schwan, Z. Naturforsch. 14b, 125 (1959).

[11] K. Asami, J. Phys. D 39, 492 (2006).

[12] P. L. McNeil and M. Terasaki, Nat. Cell Biol. 3, E124 (2001). 